\title{
EMG responses in leg muscles to postural perturbations in Huntington's disease
}

\author{
J Huttunen, V Hömberg
}

\begin{abstract}
This paper compares leg muscle electromyogram (EMG) responses to sudden toe-up tilts of a moveable platform in patients with Huntington's disease (HD), clinically normal offspring at risk of developing HD (HD risks) and healthy controls. The EMG pattern in standing subjects and patients consisted of short- and middle-latency responses (SL and ML) in the stretched triceps surae muscles and long-latency responses (LL) in the shortened tibialis anterior muscles. The SL response could be further divided into two distinct subcomponents termed SL1 and SL2. An ML response was identified in only $50 \%$ of normal subjects and patients. HD patients differed from normal subjects by showing delayed onset latencies and prolonged durations for the LL response, and smaller amplitudes for the ML response. The subjects at risk also showed diminished ML amplitudes and prolonged $L L$ durations, but normal $L L$ onset latencies. In the sitting condition, the EMG responses of the HD patients and of the HD risks did not differ from those of controls: in all groups SL1 was reduced and delayed, SL2 slightly enhanced, while $M L$ and $L L$ were absent. Because both afferent and efferent conduction times are normal in $H D$, the delayed $L L$ onset reflects abnormal supraspinal organisation of postural control in HD, and indicates that basal ganglia may have a modulatory effect on the LL responses. The normal EMG responses in the sitting patients suggest appropriate regulation of these responses according to postural set in HD.
\end{abstract}

Neurology Therapy Centre, Department of Neurology, University of Düsseldorf, Hohensandweg 37, 4000 Düsseldorf 13, Federal German Republic

J Huttunen

V Hömberg

Correspondence to: V Hömberg, Neurologisches Therapiecentrum Hohensandweg 37 D-4000 Düsseldorf 13 Federal German Republic. Received 5 December 1988 and in revised form 13 April 1989.

Accepted 27 June 1989

In subjects standing on a moveable platform, a sudden toe-up tilt evokes short- and middlelatency reflexes (SL and $M L$, respectively) in the stretched triceps surae (TS), and a longer latency response (LL) in the shortened tibialis anterior (TA) muscle. ${ }^{1} \mathrm{SL}$ and $\mathrm{ML}$ have onset latencies at about $40 \mathrm{~ms}$ and $90 \mathrm{~ms}$, respectively, and LL usually starts at about $130 \mathrm{~ms}$ in healthy subjects. While SL and ML tend to further destabilise the subject's posture, the LL response prevents the subject from falling backwards.

The latency of the SL response suggests that it corresponds to a monosynaptic myotatic or an oligosynaptic segmental reflex. The longer latency $\mathrm{ML}$ and LL components are assumed to be mediated via longer pathways. ${ }^{2}$ The ML response is enhanced in Parkinson's disease (PD), suggesting that its amplitude is modulated by basal ganglia activity. ${ }^{3}$ The LL response is delayed in upper motorneuron disease of spinal or hemispheric origin, which has led to the hypothesis of a transcortical loop mediating this response. ${ }^{4}$ However, duration and amplitude of the LL response are also changed in patients with lesions of the cerebellum, ${ }^{5}$ which therefore appears to modulate the LL responses, as well. Since the LL responses are of normal latency and amplitude in Parkinson's disease (PD), ${ }^{3}$ there is as yet no evidence for involvement of the basal ganglia in the control of these late postural adjustments.

The pathology of Parkinson's disease is characterised by degeneration of the dopaminergic input to the neostriatum. By contrast, in HD the intrinsic neurons of caudate, putamen and pallidum undergo degeneration. ${ }^{6-8}$ The most conspicious "positive" sign of $H D$, is choreatic hyperkinesia, consisting of fast and irregular movements, in contrast to the regular tremor of PD. Detailed quantitative studies of the "negative" symptoms have shown, however, that similar slowing of voluntary movement is present in both degenerative diseases of the basal ganglia..$^{910}$ In the upper extremities long-latency reflexes may be enhanced in $\mathrm{PD},{ }^{11}$ but are absent in $\mathrm{HD} .{ }^{12}$ Although postural abnormalities are common in the clinical picture of $\mathrm{HD},{ }^{13}$ no information is available about stance regulating reflexes in the lower extremities of HD patients.

The aim of this study was to analyse whether abnormalities in postural EMG responses are present in HD. The EMG responses were also recorded with the patients sitting, to discover whether the responses are appropriately regulated according to different postural sets in HD.

\section{Methods}

Twenty seven patients with definite HD (13 females, 14 males, mean age 41 , range 27 to 63 ) were studied. Demographic data of the patients are shown in table 1 . The extent of choreatic movements was assessed with a clinical score ranging from $0-3$, and the overall intellectual, social and motor impairment was evaluated with a disability score ranging from $0-3 .^{14}$ The latter is a modification of the score used by Shoulson and Fahn. ${ }^{15}$ Fourteen of the patients received sulpiride or phenothiazines, two were 
Table 1 Demographic data of HD patients

\begin{tabular}{|c|c|c|c|c|c|}
\hline Patient number & Sex & Age (years) & Disease duration (years) & Chorea grade & Disability score \\
\hline 1 & Female & 37 & 2 & $1 \cdot 0$ & $0 \cdot 0$ \\
\hline 2 & Male & 58 & 10 & $2 \cdot 0$ & $2 \cdot 0$ \\
\hline 3 & Male & 32 & 3 & $2 \cdot 0$ & $2 \cdot 0$ \\
\hline 4 & Male & 47 & 9 & 1.5 & $1 \cdot 3$ \\
\hline 5 & Male & 45 & 13 & $2 \cdot 0$ & $2 \cdot 0$ \\
\hline 6 & Male & 33 & 17 & $2 \cdot 0$ & 1.7 \\
\hline 7 & Female & 41 & 12 & 1.0 & $2 \cdot 3$ \\
\hline 8 & Female & 39 & 4 & $2 \cdot 0$ & $1 \cdot 8$ \\
\hline 9 & Female & 38 & 6 & 1.5 & $1 \cdot 3$ \\
\hline 10 & Male & 44 & 2 & 1.5 & 0.0 \\
\hline 11 & Male & 31 & 6 & $2 \cdot 0$ & $2 \cdot 0$ \\
\hline 12 & Female & 44 & 5 & $2 \cdot 0$ & 0.9 \\
\hline 13 & Female & 54 & 1 & 1.0 & 0.0 \\
\hline 14 & Female & 47 & 1 & $1 \cdot 0$ & $0 \cdot 1$ \\
\hline 15 & Male & 43 & 10 & $2 \cdot 0$ & 0.8 \\
\hline 16 & Male & 46 & 10 & $3 \cdot 0$ & $2 \cdot 2$ \\
\hline 17 & Male & 63 & 1 & $1 \cdot 0$ & 0.7 \\
\hline 18 & Female & 39 & 1 & 1.0 & $0 \cdot 1$ \\
\hline 19 & Male & 41 & 5 & 1.0 & $0 \cdot 6$ \\
\hline 20 & Female & 46 & 8 & $2 \cdot 0$ & $1 \cdot 3$ \\
\hline 21 & Male & 27 & 9 & 1.5 & $1 \cdot 3$ \\
\hline 22 & Male & 38 & 6 & $2 \cdot 5$ & $2 \cdot 4$ \\
\hline 23 & Female & 38 & 3 & 0.5 & 0.1 \\
\hline 24 & Female & 48 & 9 & $2 \cdot 0$ & $1 \cdot 3$ \\
\hline 25 & Female & 29 & 9 & 1.5 & 0.8 \\
\hline 26 & Male & 30 & 8 & 3.0 & $1 \cdot 7$ \\
\hline 27 & Female & 41 & 8 & $2 \cdot 0$ & $2 \cdot 0$ \\
\hline
\end{tabular}

on tiapride and two on the reserpine agonist tetrabenazine alone. Nine received no medication.

A control group of 26 subjects was recruited from the hospital staff. These subjects had no previous experience as subjects in neurophysiological studies and no history of neurological disease. The group consisted of 13 males and females with a mean age of 38 years (range 20 to 54).

Recordings were also made in 18 first-order offspring of $\mathrm{HD}$ patients without clinical signs or symptoms, having a $50 \%$ risk of being gene carriers of HD (HD risks). This group consisted of 10 females and 8 males with a mean age of 32 years (range 20 to 50 ).

The subjects were standing on a movable platform with their heels separated by $4 \mathrm{~cm}$ and the axes of their feet positioned at an angle of 30 degrees. The amplitude of the toe-up tilt was $4^{\circ}$ and the velocity $50^{\circ} \mathrm{s}$. Electromyographic (EMG) responses were recorded with bipolar surface electrodes placed over the lateral gastrocnemius (triceps surae) and tibialis anterior muscles bilaterally. The EMG signals were filtered with a bandpass flat from $1.6 \mathrm{~Hz}$ to $1 \mathrm{kHz}$, digitised at $2 \mathrm{kHz}$, and full-wave rectified. Eight artefact free runs were averaged for each subject.

During the recordings the subjects were instructed to stand as still as possible and to fixate on a point $1.5 \mathrm{~m}$ in front of them. The procedure was explained to the patients to minimise anxiety and choreatic movements. None of the patients showed any clear choreatic movements on the lower extremities during the reflex recordings. As EMG responses could be analysed after each trial all the trials which included bursts of involuntary EMG activity were rejected from the analysis and additional artefact free trials were added. This rarely occurred and hardly ever more than two trials out of a series of 10 had to be rejected for this reason. After two or three familiarising trials, measurement runs were performed at about 15 to $45 \mathrm{~s}$ intervals without warning the subject beforehand. Data collection was performed until eight artefact free trials had been recorded. The procedure was then repeated with the subject sitting on a chair, instructed to relax and not to respond in any way to the platform tilts. The feet were positioned as in the standing condition.

Latencies, peak amplitudes and areas of rectified EMG responses were measured with an interactive cursor display on a microcomputer. The onset of each reflex component was defined as the first rising face of EMG activity leading to a clearly defined reflex EMG activity peak. In cases where the definition of a particular reflex component appeared to be too ambiguous due to background noise the recording procedure was repeated over another eight artefact free trials. The area value of a given component was defined as the integral of amplitudes over time for the whole duration of the deflection before EMG activity reached baseline level again. This measure was found to be highly correlated with peak amplitude and duration. Stimulus onset was defined as the moment of first observable deviation of the platform position from horizontal, as recorded with a potentiometer.

As no consistent differences were observed between left and right legs, average values from the two sides were used in statistical comparisons. Significance levels between groups were computed using two-tailed Student's $t$ test for uncorrelated means, while for within-group comparisons between standing and sitting conditions the $t$ test for correlated means was used. Pearson product-moment correlation for variable pairs was used to examine the relations between reflex parameters, demographic and clinical data of the patients.

Static posturography was performed in every patient and control subject before collecting reflex data with the platform. The platform system for static recordings is equipped with four strain-gauge force transducers positioned in every corner of the platform. From the readout of these four transducers the sway path of centre of foot pressure was worked out. To 
Figure 1 Rectified and averaged EMG responses in left tibialis anterior

(TA) and triceps surae (TS) muscles to sudden dorsiflexion displacements of ankle in two normal subjects. The tick marks on the axes are separated by $10 \mathrm{~ms}$. Bottom trace shows the stimulus, which begins at 100 ms after sweep onset. Note different $y$ axis scales for different curves.

Figure 2 EMG responses to ankle displacements in two HD patients. Details as in fig 1 .
Normal 44y
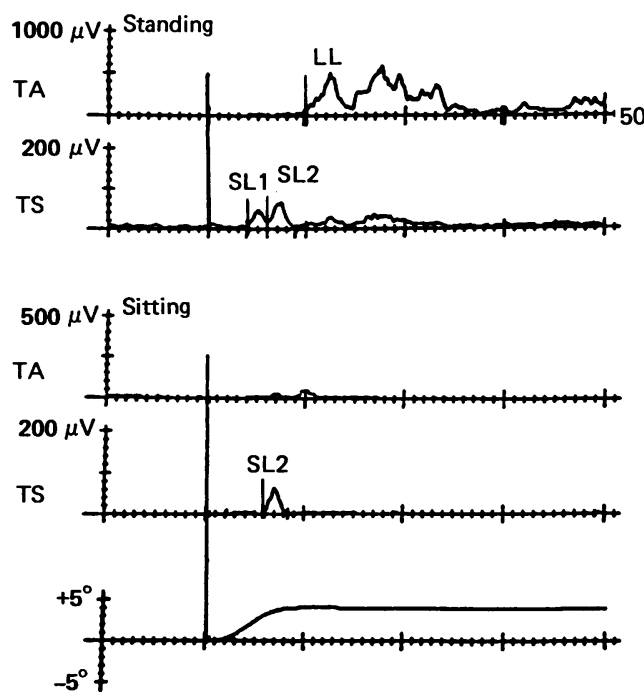

obtain sway path measurements patients and subjects were instructed to stand as still as possible fixating a point $1.5 \mathrm{~m}$ in front of them similar to the reflex recording condition. Sway path data were collected over 20 s and the total length of the sway path centre of foot pressure was determined. The dimension of this measure is $\mathrm{cm} / \mathrm{sec}$ after dividing the entire sway path length through the recording period of $20 \mathrm{~s}$.

In 25 of the HD patients magnetic cortical stimulation of motor cortex was performed to estimate the conduction time from cortex to leg muscles. Using a Cadwell MES 10 stimulator magnetic pulses (duration 70 us, intensity $2 \mathrm{~T}$ ) were delivered to the vertex region. Surface EMG responses were recorded from the abductor hallucis muscles bilaterally.

\section{Results}

Sway path measurements

The mean (SE) length of sway path for 20s for standing as still as possible in normals was 1.32 $(0.85) \mathrm{cm} / \mathrm{s}$. In HD risk patients it was slightly lower $(0.82(0.19) \mathrm{cm} / \mathrm{s})$. In HD patients sway path length was significantly longer with 3.40 $(2.87) \mathrm{cm} / \mathrm{s}$ than in normals and HD risks indicating more postural instability in the HD patients. $T$ test comparisons for uncorrelated
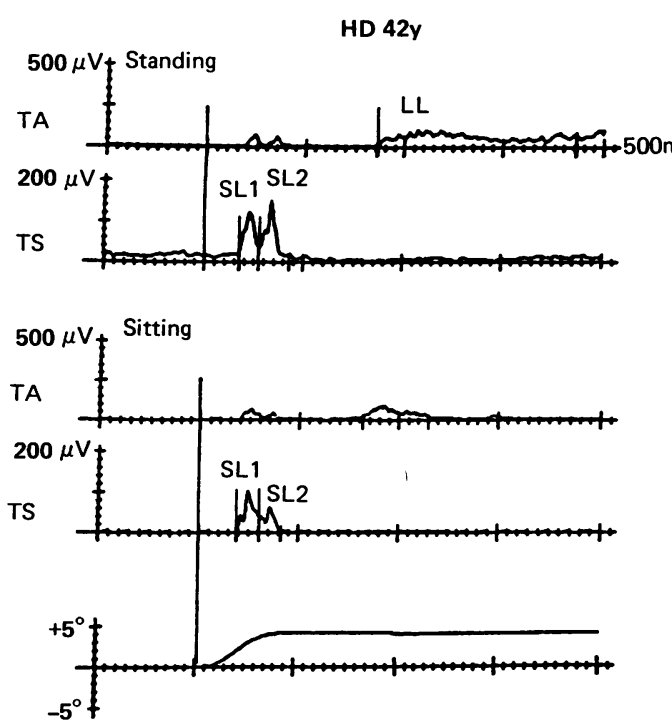

Normal 36y
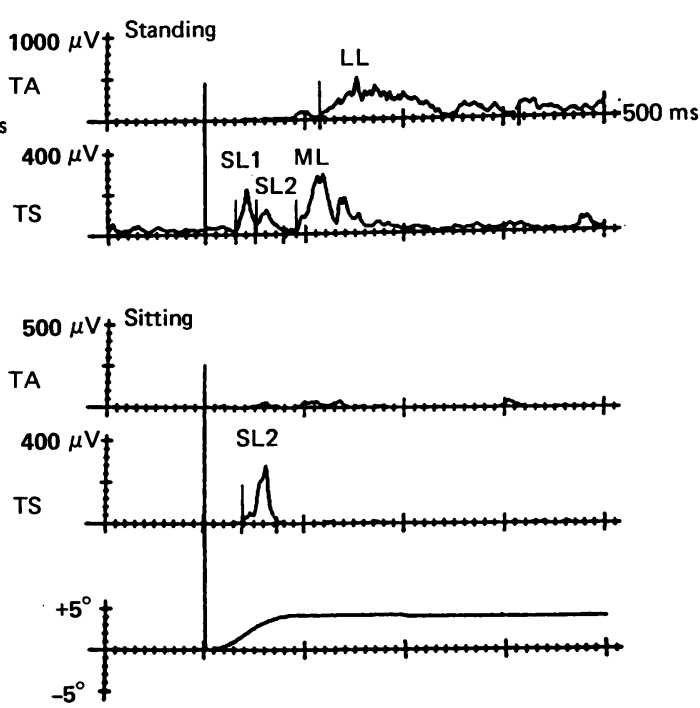

means with unequal variances showed a significant difference only between normals and HD patients $(\mathrm{t}=3.59, \mathrm{df}=34 ; \mathrm{p}<0.01)$.

\section{EMG responses in normals}

Examples of EMG responses in two normal subjects are illustrated in fig 1 . In the standing condition, slight EMG activity was always seen in the triceps surae before stimulus onset, reflecting the tonic contraction of this muscle in standing subjects. ${ }^{16}$ In tibialis anterior, no such background activity was seen. The SL reflex in triceps surae started on the average at $34 \mathrm{~ms}$ and showed only small interindividual variability (SD) $3.4 \mathrm{~ms}$. In the majority of the subjects SL consisted of two distinct peaks, which are clearly visible in the two subjects illustrated in fig 1 . Because of this consistent double-peak appearance, SL was divided into two subcomponents labelled as SL1 and SL2. The onset latency for SL2 was defined as the relative minimum of rectified EMG between the 2 peaks. Its mean value was $55 \mathrm{~ms}$, also with small interindividual variability (SD) $3.6 \mathrm{~ms}$. The peak amplitudes for SL1 and SL2 were highly variable from one subject to another, with mean values (SD) of $117(79) \mu \mathrm{V}$ and 81
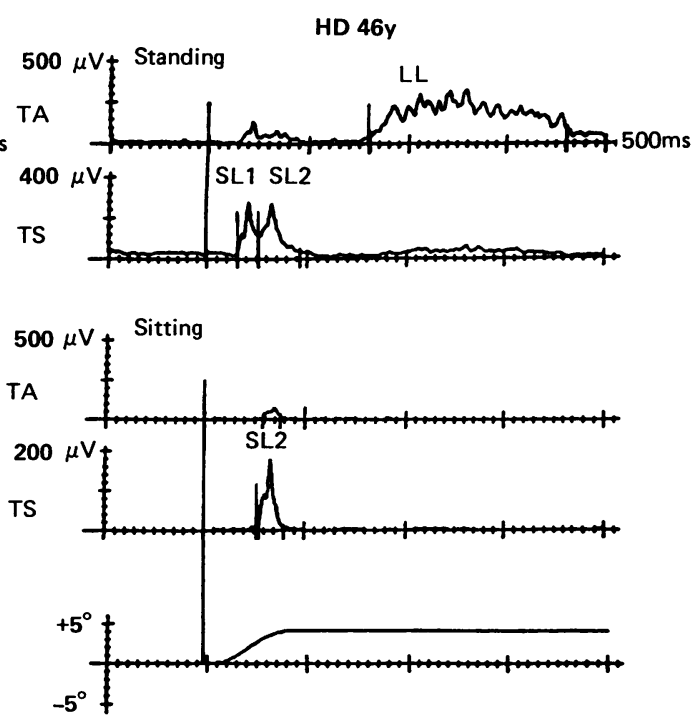
Figure 3 Mean SL1 and SL2 latencies, amplitudes and areas in normals, $H D$ risks and $H D$ patients in standing and sitting conditions. The bars denote SEM. Statistical significance levels refer to comparison between sitting and standing conditions: $\star \star=p<0.01$, $\star \star \star=p<0.001$.
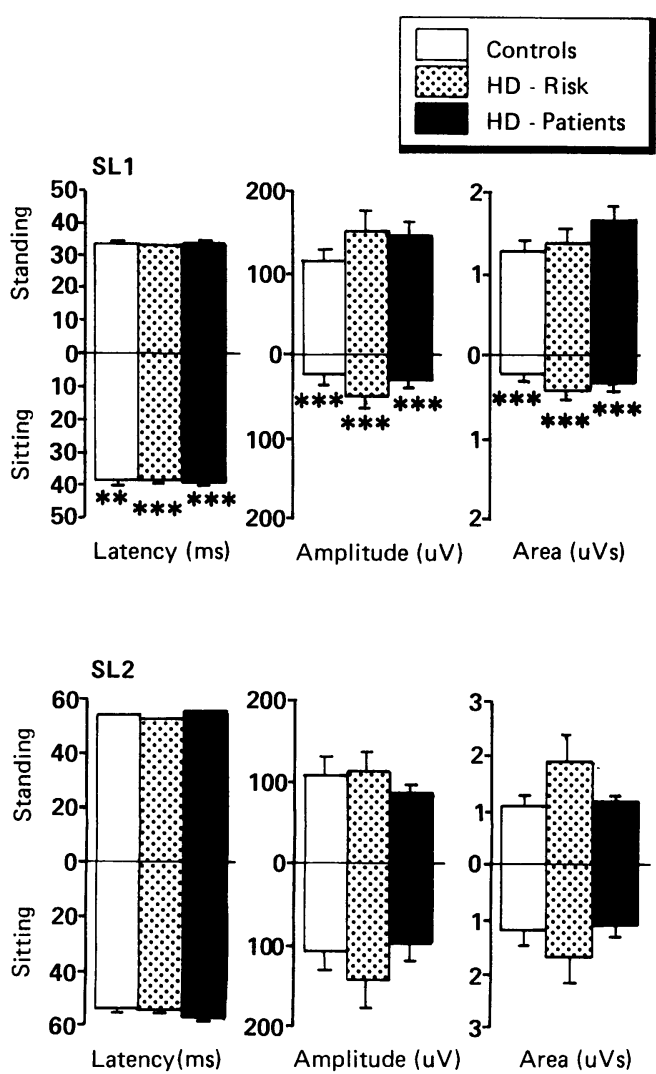

(50) $\mu \mathrm{V}$, respectively. The corresponding mean (SD) areas were $1.2(0.7) \mu \mathrm{Vs}$ and $1.3(1.0) \mu \mathrm{Vs}$. A clear ML response with an onset at $90 \mathrm{~ms}$ can be seen in the subject depicted in the right column of fig 1 , but cannot be defined for the subject in the left column. Over the entire normal population, an $\mathrm{ML}$ response was identified in either leg in 14 subjects (54\%). Its mean onset latency was $86(6) \mathrm{ms}$. The amplitude and area values showed again considerable variability with means of 144 (122) $\mu \mathrm{V}$ and $1 \cdot 8(2 \cdot 6) \mu \mathrm{Vs}$, respectively.

In tibialis anterior, a prominent $L L$ response starting at about $100 \mathrm{~ms}$ is seen in both of the subjects in fig 1 . This response was present in all subjects with a mean (SD) onset latency at 114 (16) ms. As for the earlier components, the peak amplitude and area values were variable with means of 402 (133) $\mu \mathrm{V}$ and 28 (11) $\mu \mathrm{Vs}$, respectively.

When the subjects were sitting, no background EMG activity could be seen in either muscle before stimulus onset, indicating that the subjects were properly relaxed. In contrast to the standing condition, ML and LL responses were absent in all subjects studied (fig 1). Similarly, SL1 was either abolished or considerably reduced in amplitude and delayed in onset. Both of these effects were highly significant (fig 3). The SL2 response showed distinctly different behaviour compared with SL1, being frequently enhanced in the sitting condition, as depicted in the right column of fig 1 , or remained unchanged, as illustrated in the left column. On statistical comparison, the mean latency, amplitude and area values for SL2 were not significantly different from the values obtained in the standing condition (fig 3).
EMG responses in $H D$ patients and persons at risk

In the standing condition, HD patients and persons at risk showed background EMG patterns similar to those of controls, that is, tonic activation of triceps surae but no activation of tibialis anterior. Also, SL1 and SL2 latencies and amplitudes were comparable to those of the normal subjects (fig 2), there being no statistically significant differences in any of the SL1 or SL2 parameters between the three groups (fig 3). Neither did we observe any individual patients, whose SL1 or SL2 amplitudes would have been outside the normal range of variability.

In both HD patient and HD risk groups, the frequency of occurrence of a clearly discernible ML response was similar to that found in normal subjects: an ML response in either leg was identified in nine $\mathrm{HD}$ risks $(50 \%)$ and 13 HD patients $(48 \%)$. However, statistical comparison of those patients, risks and normals with identifiable ML responses showed significantly reduced $M L$ amplitudes for $H D$ patients and HD risks (fig 4). The mean (SD) ML latencies of 89 (5) $\mathrm{ms}$ and 94 (11) $\mathrm{ms}$ in the HD risk and HD patient groups, respectively, did not differ from the mean latency for normal subjects.

Both of the HD patients in fig 2 show clearly delayed LL onset latencies when compared with the controls in fig 1 . The mean (SD) LL onset latency in the HD patient group was 139 (26) ms, being significantly higher than the value for the normals (fig 5). In contrast, the mean LL latency for the HD risk group, 115 (18) ms, did not differ from that of normals. On an individual basis, nine HD patients $(30 \%)$ showed LL latency values exceeding the mean of the normal group exceeding the confidence limit, that is, $142 \mathrm{~ms}$. Only one person at risk had a clearly delayed LL onset at $165 \mathrm{~ms}$.

The mean (SD) LL duration in HD patients was 209 (51) ms, being significantly higher than the corresponding value for normals (fig 5 ). In $13(48 \%)$ individual patients LL duration exceeded the confidence interval $(217 \mathrm{~ms})$ for the normal group. The mean (SD) LL duration

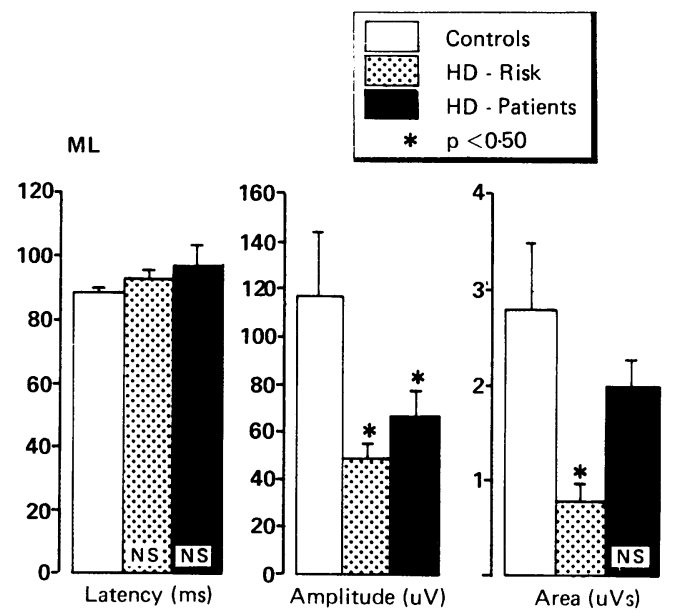

Figure 4 Mean ML latencies, amplitudes and areas in the normal group, $H D$ risks and $H D$ patients in the standing condition. $p$ - values refer to comparisons with the normal group. NS = non significant. Bars denote SEM. 
Figure 5 Means of latencies, amplitudes, areas and durations of $L L$ responses in normals, $H D$ risks and $H D$ patients in the standing condition. Details as in fig 4.
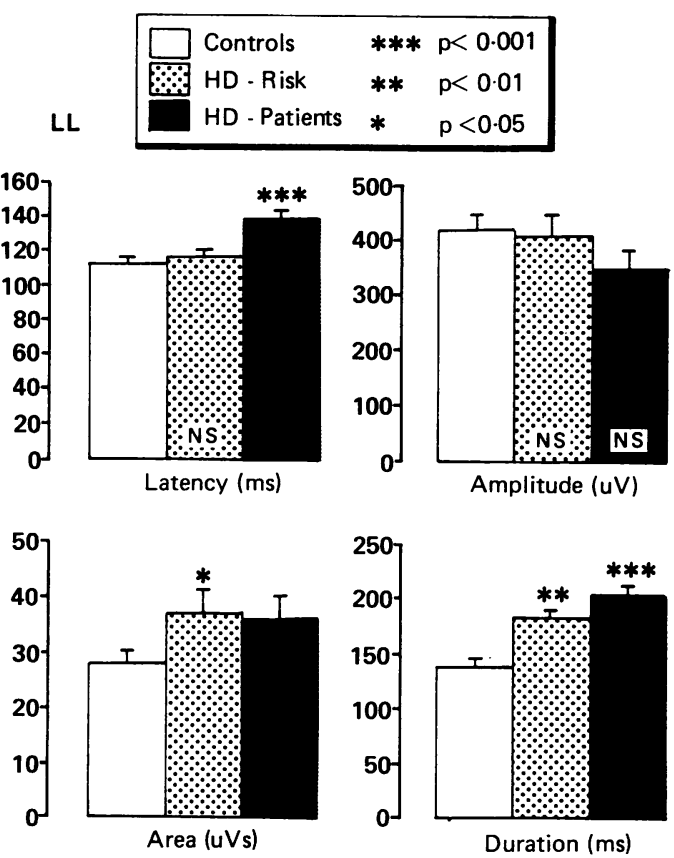

for the risk group was 184 (39) ms, also being significantly higher than for the normals group. However, only three individual risk persons showed LL durations exceeding the confidence limit for normal subjects.

The mean (SD) LL peak amplitude in HD patients was $352(183) \mu \mathrm{V}$, being slightly, but not significantly, smaller than in normals (fig 5). In the risk group LL peak amplitude was virtually identical to that of the normals subjects. The LL area values were in both HD risk and HD patient groups greater than in the normal group, reflecting the prolonged duration of the LL responses in the patients and persons at risk.

In the sitting condition, no background EMG activity could be seen before stimulus onset, indicating that the HD patients and HD risks were relaxed to a similar degree as the controls (fig 2). All EMG variables in the patients and persons at risk showed changes comparable to the changes observed in the normal group: $M L$ and LL responses were abolished in all patients, SL1 was significantly attenuated and delayed, and SL2 was not changed either in latency or amplitude. Thus, in the sitting condition statistical comparison disclosed no significant differences in any of the EMG parameters between the three groups (fig 3).

\section{Correlations of EMG variables with clinical \\ data}

Across different EMG components, only the amplitude (and area) values of SL1 and SL2 in the standing condition were significantly $(p<0.001)$ correlated with each other within all three groups $(\mathrm{r}=0.75,0.84,0.67$ in normals, HD risks and HD patients, respectively). No other significant correlations existed between any EMG parameters within any of the groups.

None of the EMG variables correlated with age in any of the three groups. In the HD patient group, the delayed LL onset latency and prolonged LL duration did not correlate with the severity of choreatic movements (table 2). Similarly, the slight positive correlations of LL latency with disease duration and disability score were insignificant. LL duration did not correlate with any of these parameters.

The delayed LL onset in HD is unlikely to be due to the medication taken by many of the patients, since three of the nine patients free of medication showed clearly delayed LL onsets.

\section{Muscle action potentials after motor cortex} stimulation

The potentials evoked in abductor hallucis muscles by motor cortex stimulation were of normal latency in all $25 \mathrm{HD}$ patients. Accordingly, no correlation existed between the muscle action potential latencies and delayed LL latency $(r=0.04)$.

\section{Discussion}

The principal finding of this study shows that LL responses in tibialis anterior, stabilising the upright posture after a sudden platform perturbation, start later and last longer in Huntington's disease compared with the normal group. The delayed LL onset is largely a group effect, but may be sufficient to distinguish individual cases from normal subjects when clearly delayed. In first-order offspring of HD patients, being at $50 \%$ risk of being gene carriers for the disease, LL onset latency appeared normal in 17 out of 18 cases, indicating that the $\mathrm{LL}$ responses do not provide a useful test to detect presymptomatic HD patients.

The ML response in TS was present in only about $50 \%$ of normals, risk persons and $\mathrm{HD}$ patients. Therefore, although showing reduced amplitudes in HD patients and in HD risks, the ML response does not provide useful diagnostic information in individual cases.

\section{Normal sequence of EMG responses in standing} and sitting conditions

For the control group, our results are in general agreement with the data reported by Diener $e t$ al, using a similar set-up. ${ }^{1-51718}$ Minor differences include shorter latencies found in the present study: The SLl onset latency (34ms) and the LL onset latency (114ms) are lower than those reported by Diener et al, while the ML onset latency of $86 \mathrm{~ms}$ falls within the range of variability found in the literature. These differences are likely to be due to

Table 2 Correlation coefficients between $L L$ parameters and demographic variables in HD patients

\begin{tabular}{llllr}
\hline & Age & Disability score & Chorea grade & Disease duration \\
\hline LL latency & 0.01 & 0.38 & -0.07 & 0.30 \\
LL amplitude & 0.17 & 0.01 & 0.26 & -0.11 \\
LL area & 0.26 & 0.03 & 0.07 & -0.16 \\
LL duration & 0.40 & 0.07 & 0.14 & 0.05 \\
\hline
\end{tabular}


different definitions of stimulus onset: Diener et al, used the signal triggering the platform tilt whereas we defined the onset as the first observable movement of the platform as recorded by a potentiometer. It therefore appears that the present latency values provide more accurate estimates for the actual time between receptor activation and muscle responses.

In the reports by Diener et al, an ML component was identified in $50-93 \% \%^{116}$ of healthy subjects. Our results agree with the lower figure; in this study identifying an $\mathrm{ML}$ response was often difficult because of its low amplitude and slow onset compared with the preceding SL1 and SL2 components. Diener $e t$ $a l^{1}$ reported that $\mathrm{ML}$ is velocity dependent: stimulus velocities exceeding the $50^{\circ} \mathrm{s}$ used in this study may be necessary to elicit this response in a larger proportion of subjects.

Another apparent difference between the present data and the data obtained by Diener $e t$ $a l$ is the dissociation of the SL response into two distinct components, SL1 and SL2. This subdivision was based on two observations: (1) SL1 and SL2 showed two distinct peaks in almost all normal subjects and HD patients, and (2) in the sitting condition SL1 and SL2 were differentially modified. It is unlikely that the SL2 component actually corresponds to an ML response, which in our recording conditions might have occurred earlier than previously reported, because a separate SL2 component could be identified also in those subjects showing a well-defined ML response. Moreover, the ML response was abolished in the sitting condition, agreeing with previous studies, ${ }^{18}$ while the SL2 component remained essentially unchanged.

Further support for the distinction between different SL1 and SL2 components can be derived from comparison with observations in the literature. In the tibialis anterior muscle Iles ${ }^{19}$ found two reflex components at 28 and $50 \mathrm{~ms}$ in response to electrical stimulation of the peroneal nerve or to mechanical tendon taps. These two responses could be functionally dissociated by their different behaviour with increasing electrical stimulus intensity. The latencies of these responses agree with the latencies of SL1 and SL2 in this study, and are both well below the value for ML. A similar occurrence of two distinct short latency reflexes was observed by Gottlieb and Agarwal $^{20}$ in the triceps surae muscle following mechanically induced dorsiflexion pulses in sitting subjects. These responses occurred at latencies of 40 and $60 \mathrm{~ms}$ comparable to our SL1 and SL2 latencies. The first of the components of Gottlieb and Agarwal ${ }^{20}$ showed a gain proportional to voluntary background activity in triceps surae whereas the second did not. In our study the triceps surae was tonically active in standing but not in sitting subjects. Therefore, our finding that only SL1 was larger in the standing than sitting condition, while SL2 remained unchanged, is in keeping with the gain characteristics described by Gottlieb and Agarwal for their short-latency components.
While the SL1 component probably corresponds to the myotatic reflex mediated via the Ia afferent system, an interpretation of the SL2 component is necessarily speculative. It might be mediated via afferents other than Ia fibres, such as group II spindle afferents, ${ }^{21}$ or it might be due to an oligosynaptically transmitted spinal Ia volley. ${ }^{22}$ As a human correlate for a group II mediated reflex is not available, ${ }^{23}$ it would be interesting to study whether SL2 fulfills criteria for group II activation, for example, by means of vibration. ${ }^{21}$ The idea that spindle II afferents become slightly less sensitive to small amplitude stretches in the presence of background fusimotor drive ${ }^{22}$ would be consistent with our findings that SL2 was slightly smaller in the standing condition, associated with background activation of the triceps surae.

At present, there is little information about the receptors being involved in the generation of ML and LL. Persistence of these responses after an ischaemic block at the ankle level causing anaesthesia of the foot and ankle, indicates that cutaneous receptors from the foot or ankle joint receptors are not involved. ${ }^{24}$

The reduction of $S L 1$ in the sitting condition may be explained as a consequence of reduced alpha-motoneuron excitability in this condition without tonic background activation. It is not known whether the same explanation applies for the abolished ML response, as well. Because the LL response was recorded in the tibialis anterior, which was not tonically activated either when standing or sitting, similar changes in the tonic background activation are unlikely to have caused the disappearance of $\mathrm{LL}$ in the sitting condition. It is therefore more appropriate to attribute the observed LL changes to different modes of central processing of afferent information according to different postural sets in the sitting and standing conditions.

\section{EMG responses in $H D$}

On clinical examination HD patients often show brisk tendon jerks. ${ }^{825}{ }^{26}$ Paulson ${ }^{25}$ even stated that hyperreflexia is the rule in $\mathrm{HD}$. However, Noth et al ${ }^{12}$ reported normal shortlatency reflexes in the upper extremities of $\mathrm{HD}$ patients. This study confirms this finding for the triceps surae muscle. These quantitative findings indicate that mono- and oligosynaptic segmental reflexes are unchanged in $\mathrm{HD}$, and that alterations in these reflexes do not contribute to the classically described hypotonia in $\mathrm{HD},{ }^{8}$ or to the increased muscle tone found in some patients. ${ }^{13}$ Since in the HD group the SL1 reflex was reduced to a similar degree in the sitting condition as in the normal group, it appears that the myotatic reflex gain is also normally regulated in HD according to varying degrees of background activation.

The tendency for the $M L$ response to be smaller in HD patients than in normals is comparable to the absence of long-latency reflex components in the upper extremities of HD patients. ${ }^{12}$ Similar parallel features for the postural ML response and the $M 2 / 3$ reflex in forearm muscles have been reported for 
patients with Parkinson's disease, in whom both types of responses are enhanced. ${ }^{311}$ The similarity of these findings indicates that the ML response may depend on mechanisms similar to the $M 2 / 3$ response in the upper extremities. The $M 2 / 3$ response has been shown to correlate with rigidity in PD. ${ }^{11}$ Hence, ML as its possible counterpart in lower extremities might be related to muscle tone regulation mechanisms, as discussed by Scholz et $a l .^{3}$ Although we did not quantitatively correlate the EMG responses with the clinical appearance of muscle tone, it would be expected that some of the 27 patients were hypertonic. None of the patients, however, showed an increased ML. Thus it is possible that the occasional hypertonia in HD may differ from classical rigidity of PD.

The finding of delayed LL onset may be considered as a neurophysiological correlate for the clinical notion of impaired postural stability in HD. Interestingly, PD, which is clinically even more characterised by impaired ability to correct postural perturbations, presents with normal LL responses. ${ }^{3}$ In PD patients, it is the exaggerated $\mathrm{ML}$ tending to assist the perturbation, which seems to correspond to postural instability. These differential findings in HD and PD suggest that different physiological mechanisms are involved in the postural impairments in the two conditions.

Both afferent and efferent conduction times in HD can be assessed with clinical neurophysiological tools: somatosensory evoked potentials after leg nerve stimulation are of normal latencies, although reduced in amplitude. ${ }^{27} 28$ Likewise, the efferent conduction time from the motor cortex to foot muscles have been found normal in HD, as assessed by magnetic cortical stimulation. ${ }^{29}$ Hence, a delay in a possible transcerebral reflex route in $\mathrm{HD}$ as documented by the increased LL latencies cannot be attributed to slowing of afferent or efferent conduction. This is in contrast to findings of delayed LL responses in cerebrovascular disease ${ }^{4}$ or multiple sclerosis, ${ }^{17}$ in which afferent or efferent conduction can be slowed down. The findings in this study indicate, however, that also in the absence of any delay in the afferent or efferent pathways a clearly delayed LL response may occur. Fig 6 shows an alignment of the fastest possible afferent and efferent conduction times in an HD patient and a normal subject, as estimated from somatosensory evoked responses to tibial nerve stimulation and muscle responses evoked by magnetic motor cortex stimulation. In the normal subject and the patient the fastest possible transmission from sensory receptors in the leg via motor cortex to leg muscles appears identical. The LL response in this HD patient, however, is considerably delayed, suggesting that in HD the LL delay depends on prolongation of intracerebral processing time. This is corroborated by the lack of correlation between LL latencies and efferent conduction times in the entire HD population, as shown for 25 patients in this study.

Prompted by the finding of normal LL responses in Parkinson's disease, Diener et $a l^{4}$
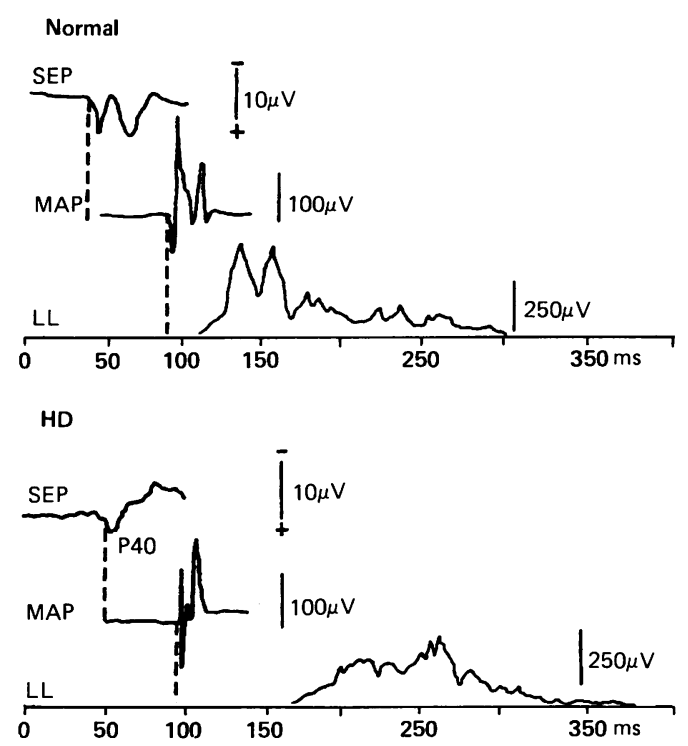

Figure 6 Somatosensory evoked potentials (SEPs) to electric stimulation of the left tibial nerve at the ankle, action potentials from the left abductor hallucis muscle after motor cortical stimulation and $L L$ responses from left $T A$ in a normal subject and an HD patient.

suggested that basal ganglia are not involved in the loop generating these responses. As atrophy of the neostriatum is the main pathological lesion in $\mathrm{HD}$, our results seem to justify a reconsideration of this conclusion. Certainly, it has to be taken into account that pathology outside the basal ganglia is involved in $\mathrm{HD}$, including the ventrobasal thalamus, ${ }^{30}$ and the cerebral cortex. ${ }^{671}$ However, the neuropathological studies demonstrating cortical atrophy in HD have mainly described subjects with advanced disease. Recent investigations on regional glucose metabolism in vivo have shown that in the early and mid-stages of the disease, no cortical hypometabolism is present. ${ }^{32-34}$ In our study, three patients with a disease duration of five years or less showed clearly delayed LL onset latencies. The most plausible explanation for these findings in early HD is that basal ganglia either belong to the loop generating LL responses or at least have a modulatory effect on the networks producing it.

It is currently assumed that the pathological mechanism underlying choreatic movements in HD is the loss of striatal gabaergic neurons. ${ }^{8}$ Because the LL latency did not correlate with severity of choreatic movements, it is possible that neurochemical factors other than the gabaergic system are responsible for postural instability in HD. Similar conclusions have been drawn previously on the basis that haloperidol treatment reduces chorea without improving the gait abnormality in HD. ${ }^{35}$

In PD, Diener et $a l^{17}$ reported persistence of $M L$ and LL responses in the sitting condition, which was interpreted as an exaggerated shortening reaction in PD, as shown earlier for upper extremity muscles, ${ }^{36}{ }^{37}$ as well as for the tibialis anterior ${ }^{38}$ Diener et $a l^{17}$ concluded that in PD the regulation of postural response according to different functional demands is impaired. Our finding demonstrates a lack of a 
similar appropriate regulation of the leg EMG responses according to postural set in $\mathrm{HD}$.

This study was supported by a scholarship from the Alexander von Humboldt Foundation and by grants from the Deutsche Forschungsgemeinschaft (SFB 200, B9). We thank Dr H W Lange for referring his HD patients to us.

1 Diener HC, Dichgans J, Bootz F, Bacher M. Early stabilisation of human posture after a sudden disturbance: influence of rate and amplitude of displacement. Exp Brain Res 1984a;56:126-34.

2 Diener HC, Dichgans J. Long loop reflexes and posture. In: Bless $\mathrm{W}$, Brandt $\mathrm{T}$, eds. Disorders in Posture and Gait. Amsterdam: Elsevier 1986:41-51.

3 Scholz E, Diener HC, Noth J, Friedemann H, Dichgans J, Bacher M. Medium and long latency EMG responses in leg muscles: Parkinson's disease. J Neurol Neurosurg Psychiatry 1987;50:66-70.

4 Diener HC, Ackermann H, Dichgans J, Guschlbauer B. Medium- and long-latency responses to displacements of the ankle joint in patients with spinal and central lesions. Electroenceph Clin Neurophysiol 1985;60:407-16.

5 Diener HC, Dichgans J, Bacher M, Guschlbauer B. Characteristic alterations of long-loop "reflexes" in patients with Friedreich's disease and late atrophy of the cerebellar anterior lobe. J Neurol Neurosurg Psychiatry 1984;47: 679-85.

6 Lange H, Thörner A, Hopf A, Schröder KF. Morphometric studies of the neuropathological changes in choreatic studies of the neuropathological chang
diseases. J Neurol Sci 1976;28:401-25.

7 Bruyn GW, Bots GThAM, Dom R. Huntington's chorea: current neuropathological status. In: Chase TN et al, eds. Advances in Neurology. New York: Raven Press, 1979: 83-93.

8 Martin JB. Huntington's disease: new approaches to an old problem. Neurology 1984;34:1059-72

9 Hefter H, Hömberg V, Lange HW, Freund H-J. Impairment of rapid movement in Huntington's disease. Brain 1987;110:585-612.

10 Thompson PD, Berardelli A, Rothwell JC, Day BL, Dick JPR, Benecke R, Marsden CD. The coexistence of bradykinesia and chorea in Huntington's disease and its implications for theories of basal ganglia control of movement. Brain 1988;111:223-44.

11 Tatton WG, Lee RG. Evidence for abnormal long-loop reflexes in rigid Parkinsonian patients. Brain Res

12 Noth J, Podoll K, Friedemann H-H. Long-loop reflexes in small hand muscles studied in normal subjects and in patients with Huntington's disease. Brain 1985;108: 65-80.

13 Hayden MR. Huntington's Chorea. Berlin Heidelberg, New York: Springer, 1981.

14 Lange HW, Strauss W, Hassel PC, Wöller W, Tegeler J. Langzeittherapie bei Huntington-Kranken. Psycho 1983;5:286-90.

15 Shoulson I, Fahn S. Huntington disease: clinical care and evaluation. Neurology 1979;29:1-3.

16 Smith JW. The forces operating at the human ankle joint during standing. $J$ Anat 1957;91:545-64.

17 Diener HC, Dichgans J, Hülser P-J, Büttner U-W, Bacher $M$, Guschlbauer B. The significance of delayed long-loop responses to ankle displacement for the diagnosis of multiple sclerosis. Electroenceph Clin Neurophysio 1984b;57:336-42.

18 Diener C, Scholz E, Guschlbauer B, Dichgans J. Increased shortening reaction in Parkinson's disease reflects difficulty in modulating long loop reflexes. Movement Difficulty in modulating

19 Iles JF. Responses in human pretibial muscles to sudden stretch and to nerve stimulation. Exp Brain Res 1977;30:451-70.

20 Gottlieb GL, Agarwal GC. Response to sudden torques about ankle in man: myotatic reflex. $J$ Neurophysio 1979;42:91-106.

21 Matthews PBC. Evidence from the use of vibration that the human long-latency stretch reflex depends upon spindle secondary afferents. $J$ Physiol 1984;348:383-415.

22 Matthews PBC. Mammalian muscle receptors and their central actions. London: Edward Arnold, 1972.

23 Delwaide PJ, Olivier E. Pathophysiological aspects of spasticity in man. In: Benecke R, Conrad B, Marsden CD ed. Motor Disturbances I. London: Academic Press 1987:153-67.

24 Diener HC, Dichgans J, Guschlbauer B, Mau H. The significance of proprioception on postural stabilisation as assessed by ischemia. Brain Res 1984;296:103-9.

25 Paulson GW. Diagnosis of Huntington's disease. In: Chase et al, eds. Advances in Neurology, vol 23. New York: Raven Press 1975:177-84.

26 Bird ED. Chemical pathology of Huntington's disease. Ann Rev Pharmacol Toxicol 1980;20:533-51.

27 Noth J, Engel L, Friedemann H-H, Lange HW. Evoked potentials in patients with Huntington's disease and their offspring. I. Somatosensory evoked potentials. Electroenceph clin Neurophysiol 1984;59:134-41.

28 Bollen EL, Arts RJ, Roos RA, Van der Velde EA, Burum OJ. Somatosensory evoked potentials in Huntington's ch. Somatosensory evoked potentials in Huntington's

29 Thorear

A, Kachi T, Marsden CD. Electrophysiology of the A, disorders. Movement Disorders 1986;1:113-7.

30 Dom R, Malfroid M, Baro F. Neuropathology of Huntington's chorea. Neurology 1976;26:64-8.

31 Lange HW. Quantitative changes of telencephalon diencephalon, and mesencephalon in Huntington' chorea, postencephalitic, and idiopathic parkinsonism. Verhandlungen der Anatomischen Gesellschaft 1981, 75:923-5.

32 Kuhl DE, Phelps ME, Markham CH, Metter EJ, Riege WH, Winter J. Cerebral metabolism and atrophy in Huntington's disease determined by $18 \mathrm{FDG}$ and computed tomographic scan. Ann Neurol 1982;12:425-34.

33 Hayden MR, Martin WRW, Stoessl AJ, Clark C,

Hollenberg S, Adam MJ, Ammann W, Harrop R, Rogers J, Ruth T, Sayre C, Pate BD. Positron emission tomography in the early diagnosis of Huntington's disease. Neurology 1986;36:888-94.

34 Young AB, Penney JB, Starosta-Rubinstein S, Markel DS Berent S, Giordani B, Ehrenkaufer R, Jewett D, Hichwa $R$. PET scan investigations of Huntington's disease: cerebral metabolic correlates of neurological features and functional decline. Ann Neurol 1986;20:296-303.

35 Koller WC, Trimble J. The gait abnormality of Huntington's disease. Neurology 1985;35:1450-4.

36 Andrews CJ, Burke D, Lance JW. The response to muscle stretch and shortening in parkinsonian rigidity. Brain 1972;95:795-812.

37 Angel RW. Shortening reaction in normal and parkinsonian subjects. Neurology 1982;32:246-51.

38 Berardelli A, Hallett M. Shortening reaction of human tibialis anterior. Neurology 1984;34:242-5. 